\title{
A QUESTION OF LASER INTENSITY
}

\author{
G.A. McRAE \\ AECL Research, Chalk River Laboratories \\ Chalk River Ontario, Canada K0J 1J0
}

(Received 1 May, 1993)

\begin{abstract}
Parameterization of laser intensity requires different practical definitions which depend on the temporal and spatial characteristics of the optical beam. Comparison of observations obtained using different lasers, with similar numerical values of intensity, will prove misleading for non-linear phenomena. The invariant quantities that each experiment should have in common are the determinable model parameters.
\end{abstract}

KEY WORDS: Laser Intensity Profiles, Laser Intensity

\section{INTRODUCTION}

Although the intensity (energy/area/time) of an electro-magnetic wave is generally a well understood quantity, it is not always reported in a consistent way for laser experiments. It is not uncommon to find reports of experimental observations, obtained with powerful lasers operating at, apparently, the same intensity, displaying quite disparate claims. Some of this discrepancy can be attributed to inconsistencies in the calculation of the intensity for lasers with different spatial and temporal beam geometries. Further complications may arise when trying to reconcile the intensities quoted in $a b$ initio studies, which are often based on plane waves, with those of experimentalists, who generally work with Gaussian beams.

\section{THEORY}

The intensity of a laser pulse travelling with speed $c$, in the $+z$ direction, with a temporal profile given by $f(z-c t)$, can be parameterized by

$$
I=I_{0} f(z-c t) g(r)
$$

where $g(r)$ is the profile in the radial direction and $I_{0}$ is the maximum intensity value, sometimes called the intensity at beam center.

Usually, the intensity of the beam is not directly measured. Instead, the beam is characterized by a measurement of the temporal and radial beam profiles along with the total energy. The energy in the pulse is measured by placing a detector, 
with a large cross-sectional collection area $(A)$, at some distance $z$ and integrating the intensity over time.

$$
E=\iint_{-\infty}^{+\infty} I d t d A
$$

This measurement puts constraints on the definition of $I_{0}$ in equation 1 .

As a first example, consider a light beam that has both a radial and temporal 'top hat' profile, defined such that the intensity is constant $\left(I=I_{0}\right)$ for some duration $\tau_{t}$ and for a radial distance $r$ less than some $r_{t}$, and zero otherwise. In this instance, the constraint of equation 2 yields

$$
I_{0}=\frac{E}{\pi r_{t}^{2} \tau_{t}}
$$

A different expression for $I_{0}$ is obtained if the temporal or spatial profile of the laser pulse is different. As a second example, consider a radial top hat, as above, and a Gaussian-shaped temporal pulse. The time dependent part of the intensity can be expressed as

$$
\exp \left[\frac{-2(z-c t)^{2}}{(c \tau)^{2}}\right]
$$

where $\tau$ is the time at which the intensity falls to $1 / e^{2}$ of its maximum value. A change of variables allows the time integration in equation 2 to be written as

$$
\frac{\tau}{2} \int_{-\infty}^{+\infty} \exp \left(-u^{2}\right) d u=\tau \sqrt{\frac{\pi}{2}}
$$

Including the integration over the area of the radial top hat and solving for $I_{0}$ yields

$$
I_{0}=\frac{\sqrt{2} E}{\pi^{3 / 2} r_{t}^{2} \tau}
$$

The third and final example is a beam with both a transverse and a temporal Gaussian profile

$$
I=I_{0} \exp \left[\frac{-2(z-c t)^{2}}{(c r)^{2}}-\frac{2 r^{2}}{r_{0}^{2}}\right]
$$

where $r_{0}$ is the radius at which the intensity falls to $1 / e^{2}$ of its maximum value at some time $t$. The area integration requires solution of

$$
2 \pi \int_{0}^{r_{c}} \exp \left(-2 r^{2} / r_{0}^{2}\right) r d r
$$

where $r_{c}$ is the radius of the interaction region, often defined by some reaction cell wall. This integral can be solved to give

$$
\frac{\pi r_{0}^{2}}{2}\left[1-\exp \left(-2 r_{c}^{2} / r_{0}^{2}\right)\right]
$$


For most studies $r_{c} \gg r_{0}$ and the second term can be ignored. Combining this with the result of the temporal integration over the Gaussian profile (Eq 5) and solving for $I_{0}$ in equation 2 yields

$$
I_{0}=\frac{2 \sqrt{2} E}{\pi^{3 / 2} r_{0}^{2} \tau}
$$

Equations 3, 6 and 10 show that $I_{0}$ is defined differently depending on the beam shape, because of the requirement that the functional form of the beam profile be normalized to the energy in the pulse. It is not correct simply to substitute these different $I_{0}$ 's into intensity-dependent formulae, because $I_{0}$ does not give a unique representation of an experimental situation, since the same value of $I_{0}$ can be obtained in an infinity of ways by varying $E$ along with the radial and temporal dimensions.

\section{PARAMETERIZING OBSERVABLES}

An intensity-dependent physical observable will implicitly be averaged over the spatial and temporal profile of an interrogating laser beam, within the volume of the observation cell. A useful example of parameterization is $m$-photon ionization through virtual states. The rate of ion production varies as $\sigma_{m} I^{m}$, where $\sigma_{m}$ is a generalized cross-section. The volume-averaged ion yield can be expressed as

$$
S_{m}=\frac{n}{V_{c}} \iint \sigma_{m} I^{m} d t d V
$$

where $\boldsymbol{n}$ is the number of atoms or molecules initially in some reaction cell of length $L$ and volume $V_{c}=\pi r_{c}^{2} L$.

For a radially cylindrical (top hat) pulse with a Gaussian temporal profile, equation 11 becomes

$$
{ }^{t} S_{m}=\left(r_{t}^{2} / r_{c}^{2}\right) n \sqrt{\pi /(2 m)} \tau \sigma_{m} I_{0}^{m}
$$

where $I_{0}$ is given by equation 6 (when $r_{c}>r_{t}$, otherwise $r_{t}=r_{c}$ ).

For the same Gaussian-temporal profile, but now with a Gaussian radial intensity distribution, equation 11 becomes

$$
{ }^{g} S_{m}=\left(r_{0}^{2} / r_{c}^{2}\right) n \sqrt{\pi /\left(8 m^{3}\right)} \tau \sigma_{m} I_{0}^{m}
$$

where $I_{0}$ is given by equation 10 .

For a linear process $(m=1)$ there is no difference in the ion signals for the top hat and Gaussian beam geometries if the total energy in both beams is the same. The ratio

$$
\frac{{ }^{t} S_{m}}{{ }^{g} S_{m}}=\frac{2 m}{2^{m}}\left(\frac{r_{0}}{r_{t}}\right)^{2 m-2}\left(\frac{E \text { tophat }}{E \text { gaussian }}\right)^{m}
$$

is unity for a linear process and equal energy beams. This result is quite general.

Comparison of equations 12 and 13 shows the difficulty inherent in thinking in terms of intensity. Two experimenters working with the same number of molecules 


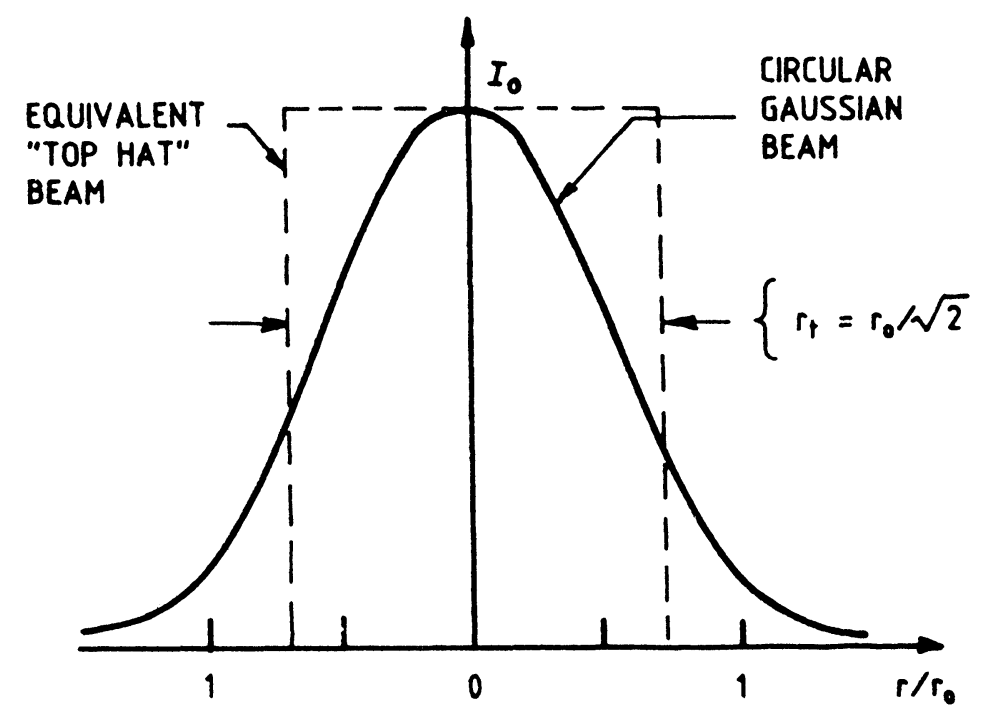

Figure 1: The equivalent radial top hat beam (same value of $I_{0}$ ) for a cylindrical Gaussian beam of radius $r_{0}$.

in the same cell could see different ion yields for intensities defined by the same numerical values of $I_{0}$. As an example, consider the ratio of expected ion signals from a laser beam with a Gaussian radial profile compared to the ion signal from an "equivalent" top hat beam. The radius and area of a top hat beam, with the same peak intensity and total power as a cylindrical Gaussian beam, are $r_{t}=r_{0} / \sqrt{2}$ and $\pi r_{0}^{2} / 2$ (see Figure 1). The ratio of the equivalent top hat to Gaussian ion signals varies simply as

$$
\frac{{ }^{t} S_{m}}{{ }^{s} S_{m}}=m
$$

The value of $m$ can be on the order of 10's for multiphoton ionization studies. In this instance, an order of magnitude difference in ionization yield could be reported for the same peak intensities!

Two experimenters, working with a non-linear process and with different shaped laser pulses, will not be able to compare intensities directly and plots of ion yield versus $I_{0}$ will be quite different. Comparison of the observables of any non-linear process, based solely on intensity, should prove difficult. For instance, threshold intensities, or, intensities for $50 \%$ decomposition, will be laser-dependent and will not be overly helpful to colleagues with different laser-beam geometries.

The one thing that everyone should have in common is the value of the crosssection, $\sigma_{m}$. Comparisons between experiments can be made only on the values obtained for the determinable parameters, in this example the $\sigma_{m}$. At the same 
time, it should be clear that simply approximating a Gaussian by a top hat, or visa versa, will result in grossly distorted values of the determinable parameters for highly non-linear processes.

\section{CONCLUSION}

Parameterizing experimental data, for pulsed laser experiments, in terms of some maximum intensity $I_{0}$ alone is not sufficient. A correct method requires an integration, over the interaction volume, of the intensity-dependent functional form prescribed for the observable, in which the intensity distribution has been properly normalized to the energy in the laser beam. This procedure takes into account the beam shape and results in a parameterization in terms of an $I_{0}$, defined by the laser temporal and geometrical profile, which will be different for different lasers. Comparison of observations obtained with lasers with different profiles, but similar numerical values of $I_{0}$, will be misleading. In the end, experiments with different laser geometries will all yield the same value of some determinable interactionparameter: for instance a cross-section, $\sigma_{m}$. 